\title{
Free-Living Physical Activity Monitoring in Adult US Patients with Multiple Sclerosis Using a Consumer Wearable Device
}

\author{
Pronabesh DasMahapatra $^{a} \quad$ Emil Chiauzzi $^{a} \quad$ Rishi Bhalerao $^{a}$ \\ Jane Rhodes ${ }^{\text {b }}$ \\ a PatientsLikeMe Inc., Cambridge, MA, USA; ${ }^{b}$ Biogen Idec, Cambridge, MA, USA
}

\section{Keywords}

Activity monitors - Biaxial/triaxial accelerometer devices - Conventional wearable devices . Digital devices · Mobility · Multiple sclerosis · Objective data - Patient-reported outcomes .

Wearable physical activity monitoring

\section{Abstract}

Introduction: Wearable devices have been used to characterize physical activity in multiple sclerosis (MS). The objectives of this study were to advance the literature on the utility of freeliving physical activity tracking from secondary analyses of a pilot study in MS patients. Method: The original observational study was conducted in participants with MS at PatientsLikeMe (www.PatientsLikeMe.com), an online network of patients with chronic diseases. Participants completed a baseline self-assessment, and received a Fitbit One ${ }^{T M}$ wearable device with instructions to upload data. Eligible participants (1) self-reported MS, (2) logged on to the PatientsLikeMe website 90 days prior to enrollment, and (3) consented to participate electronically. Participants (1) < 18 years, (2) living outside the United States, and (3) requiring wheelchair assistance for most daily activities were excluded. The secondary analyses were limited to participants with complete data on MS type, disease duration, and Multiple Sclerosis Rating Scale (MSRS) and at least 7 days of wearable data. Step count was used as a measure of physical activity. Results: The analysis cohort of 114 participants uploaded a mean of 20.1 days of wearable data over the 23-day study (87\% adherence); participants averaged 4,393 steps per day. The mean age of participants was 52 years, predominantly female (75\%), relapsing-remitting type (79\%), with mean disease duration of 16 years. Mean MSRS score within 30-day of baseline was $32 ; 72 \%$ reported mild-moderate walking disability. The reliability of step count measured by intraclass correlation was 0.55 for a single day, $\geq 0.7$ for 2 -day average, and $\geq 0.9$ for 7-day average. After controlling for covariates, self-reported disease severity (MSRS quartile) was an independent predictor of step count $(p<0.001)$. Least square means (LS means) 
DasMahapatra et al.: Free-Living Wearable Activity Monitoring in Multiple Sclerosis

for participants that were least disabled (lowest quartile) was 5,937 steps, which was significantly higher than participants in the second, third, and fourth quartiles $(4,570,3,490$, and 3,272 , respectively). Similarly, LS means of participants with no ambulatory disability (measured by MSRS walk component) was 6,931 steps, significantly higher than participants with greater disability $(4,743,4,394,2,727$ steps for symptomatic, mild, and moderate disability, respectively, $p<0.001)$. Discussion: Using an interactive platform, this study captured freeliving mobility data in MS patients. Important metrics such as the use of a minimum of 2-day estimates and self-reported disability were found to be robust indicators and correlates, respectively, of participant activity levels. Further triangulation of such metrics may reduce the burden on patients, clinicians, and researchers when monitoring clinical status.

(C) 2018 The Author(s)

Published by S. Karger AG, Basel

\section{Introduction}

Digital technology has been used regularly by patients and practitioners to facilitate disease and symptom management [1]. More recently, the landscape of digital health technology has expanded to mobile health, health information technology, telehealth, telemedicine, and medical and consumer-grade devices [2]. Such technologies enable providers to capture meaningful information about each patient, which facilitate data-driven individualized assessment and management. These technologies also enable patients and consumers to efficiently manage and track their health- and wellness-related activities.

Among available technologies, wearable activity monitors have been widely adopted for health and wellness monitoring [3]. Due to the ease of access to free-living mobility data in a real-world environment, wearable activity monitors may provide insight into the progression and impact of illnesses $[4,5]$. Studies have shown that activity monitors can detect differences in mobility between ostensibly healthy elderly or adult populations relative to elderly with reduced mobility [6]. With the advancement in wearable technology, devices can passively and objectively measure high-resolution mobility data, as opposed to subjective questionnaires and observations which are subject to bias of self-reporting or shorter duration of formal testing $[7,8]$. This has led to increasing research on consumer activity monitors. A targeted PubMed database search limited to publications on wearable devices manufactured by Fitbit ${ }^{\mathrm{TM}}$ in 2017 identified over a hundred articles. The studies cover three broad core topics: (1) the validity, reliability, and usability of activity monitors to measure health states in healthy and disease populations, (2) the utility in generating clinical relevant data at the point of care, (3) integration into health promotion, disease prevention, and behavior change programs. With respect to validity and reliability, studies have demonstrated acceptable measurement properties of activity monitors [9], although limitations were noted in populations with low walking speed [10]. User acceptance of consumer activity trackers is high as a result of the technological improvements in functionality, appeal, and form factor $[11,12]$. As for the impact at point of care, a recent study by Birkeland et al. [13] illustrated that wearable device-determined step count was able to determine treatment effect in patients with coronary microvascular dysfunction. The specific role of activity monitors at the point of clinical care has been reported in oncology, COPD, and other chronic medical conditions [8]. Further, a pooled analysis of randomized trials that includes activity monitor-based counselling reported significant improvements in several health markers (hemoglobin A1c, systolic blood pressure, and body mass index) in patients with type 2 diabetes [14].

Accordingly, there has been increasing attention on the use of consumer activity monitoring devices with multiple sclerosis (MS). MS is a disease characterized by damage to the myelin sheath that encapsulates neuronal projections in the central nervous system, causing 
transient or progressive debilitating symptoms and disability including ambulatory difficulties. Digital markers of MS have been extensively studied for symptom tracking, wearables such as accelerometers, gyroscopes, eye-trackers, grip sensors, and multisensors [15]. In other neurological diseases such as Parkinson disease, stroke, dementia, traumatic brain injury, and ataxia, studies have shown associations between activity tracking devices and other mobility assessments [16]. The importance of mobility in MS is irrefutable both as an assessment of overall health status and a component of disease management [17].

In MS, decreased ambulatory activity assessed via remote monitoring is associated with greater disability and lower quality of life [18]. Studies have shown ambulatory monitoring to be a reliable and valid outcome measure of activity levels in people with neurological diseases [19]. In MS patients, the number of daily steps has been shown to be a reliable and valid indicator of free-living walking behavior [20]. MS patients that are male, unemployed, have a high school education or less, and experience MS that is progressive, with higher levels of disability, or of longer duration take fewer steps per day [21]. Lower daily step counts are associated with greater impairment as measured by the Expanded Disability Status Scale (EDSS) and are moderately correlated with other measures of walking behavior such as oxygen cost of walking, the six-minute walk test, Multiple Sclerosis Walking Scale (MSWS), and Patient Determined Disease Steps (PDDS) scale [18, 22]. Clinically meaningful changes as measured by 1-point change in PDDS and 10-point change in MSWS can be detected in as little as a change of 800 steps per day [22].

Activity-monitoring devices that objectively record free-living walking behavior via motion sensors worn on the body may be a potential metric for quantifying ambulation and overall health status of MS patients [4], but the state of the field should be currently regarded as in a validation phase [18]. There is a continued need for more evidence of usage in realworld settings; larger samples, broader functional ranges, and appropriate metrics to gain greater confidence in the use of activity monitors in tracking and managing MS. For example, Norris et al. [23] found that to calculate a reliable mean daily step count in unaided walking, a minimum of any random 2-day combination is required. However, that study was conducted with a relatively small sample of MS patients that were not highly functioning.

To understand the real-world usage of commercially available activity-monitoring devices, this study is a secondary analysis of data obtained from PatientsLikeMe (www.PatientsLikeMe. com) study with members of an MS online community. Utilizing data from an earlier feasibility study of wearable activity tracking with MS, this study involved a secondary analysis to: (1) examine the reliability of physical activity data obtained from consumer-grade activity monitors in a free-living environment; (2) characterize participants based on activity patterns by age, gender, disease duration, and MS type; (3) explore the relationship between the Multiple Sclerosis Rating Scale (MSRS, a patient-reported disease severity measure) and the number of steps captured by a wearable activity monitor; and (4) compare age-related change in activity in the participant cohort relative to the general population and other disease cohorts.

\section{Materials and Methods}

\section{Original Observational Study}

Participants

In the present investigation, secondary analyses were derived from an earlier observational study in which participants were enrolled from the PatientsLikeMe member community. More information on the original study has been described elsewhere [24]. The following section will describe the original study briefly, and the subsequent sections will describe the secondary analysis that is the subject of this paper. 
DasMahapatra et al.: Free-Living Wearable Activity Monitoring in Multiple Sclerosis

PatientsLikeMe is an online research platform, designed to allow platform members to share data about their conditions, treatments, symptoms, and comorbidities through structured data collection, but with some features of an online social network [25]. Members join the site with the understanding that they may be periodically invited to participate in research studies. The concordance of patient-reported data shared by MS patients on the website with medical claims data has been investigated and reported in an earlier publication [26].

The original observational study was conducted in June and July 2014. Participants reporting MS on their online profiles were recruited and consented, and provided with a consumer-grade activity monitor (Fitbit One ${ }^{\mathrm{TM}}$; www.fitbit.com) for capturing their physical activity over an observational period of 3-4 weeks. The study recruitment was noteworthy in terms of speed: the cohort of 203 patients was recruited online in just over a day from a pool of highly engaged participants. In addition to physical activity data, this cohort self-reported their medical history on the PatientsLikeMe platform.

Procedures

Informed Consent. Participants who fit the screening criteria were directed to an informed consent page, where a written statement of research information with the informed consent was presented. The statement of research information was also sent to participants when they received their wearable devices by postal mail. The research protocol received ethical approval by the New England Institutional Review Board (NEIRB) on June 6, 2014.

Activity Monitoring. Fitbit One ${ }^{\mathrm{TM}}$ devices were mailed to participants with instructions on activation and authorization of data sharing between the manufacturer and PatientsLikeMe. This authentication granted research personnel permission to access their real-time device data. The device automatically transmits activity data to Fitbit servers when in proximity of the patient's Bluetooth ${ }^{\circledR}$-enabled computer or smartphone. Daily data were downloaded from Fitbit through their public web service using an application program interface. Participants were asked to wear the devices during their daily activities and charge the devices as needed. Daily step count data were collected for all participants. The Fitbit One ${ }^{\mathrm{TM}}$ has been shown to be a valid and reliable indicator of step counts $[15,16]$.

Patient Communications. A guide for participation was sent to participants via email and included in print form with their device shipments. Instructions included a stepwise guide on how to authorize and upload data. The authorization process required patients to log on the website and activate a data sharing button which permitted the investigators to obtain Fitbit data via the manufacturers application program interface. The uploading process transferred their activity data to a dashboard using Bluetooth Low Energy (BLE) technology which sync with phones, tablets, and certain computers (some computers required a Fitbit dongle to be plugged in for uploads). Users were sent periodic email reminders throughout the study period. These emails included information on how to use the devices, instructions on how to authenticate the device, contact information for study personnel, as well as reminders to update MSRS on their PatientsLikeMe profiles. This study established a framework for deploying an activity tracker for longitudinal data collection, $80 \%$ of participants were able to authorize data sharing and upload data.

Measures

Data used for analyses were collected via electronic surveys at baseline, the PatientsLikeMe platform, and from Fitbit $\mathrm{One}^{\mathrm{TM}}$. The measures for our analyses are described below.

Patient Characteristics. Demographic information (age, gender) and MS-specific characteristics (MS type, disease duration) were obtained from their PatientsLikeMe profiles upon study enrollment. 
DasMahapatra et al.: Free-Living Wearable Activity Monitoring in Multiple Sclerosis

Multiple Sclerosis Rating Scale. The MSRS is an instrument that measures functional disability in MS and includes seven items that assess walking, upper limb function, vision, speech, swallowing, thinking/memory/cognition, sensation/burning/pain (Appendix). The properties of the instrument have been validated and published elsewhere [27]. For analyses, we used data from participants that reported MSRS within 30 days of the baseline assessment.

Device Data. Fitbit One ${ }^{\mathrm{TM}}$ Activity Monitoring device, manufactured by Fitbit, San Francisco, CA (http://www.fitbit.com/), was used to measure physical activity. The device clips on to an individual's belt, pocket, or bra and contains a 3-axis accelerometer that determines threshold of steps based on motion patterns that are most indicative of people walking. The device has wireless automatic syncing features compatible with smartphone, tablet, or computer and has a battery life of approximately 14 days. The following information was collected from participants using the Fitbit One ${ }^{\mathrm{TM}}$ device: (1) activation/authentication of the device, (2) number of steps taken per day, (3) distance, (4) calories burned per day (based on self-reported data), (5) "active" min - time of day, days of week (calculated for activities at or above about 3 metabolic equivalents after $10 \mathrm{~min}$ of continuous moderate-to-intense activity). (6) sleep (duration of sleep, number of awakenings throughout the night), (7) floors, and (6) timestamp and dates of readings. For our secondary data analyses, step count was used as the main proxy outcome for physical activity.

\section{Secondary Data Analysis}

Participant Selection for Secondary Analysis

Participant data for this study were drawn from the existing data set generated by the feasibility study mentioned above. The inclusion criteria in the observational study were: (1) participants self-reporting MS as a condition on their PatientsLikeMe profile, (2) $\geq 1$ PatientsLikeMe website login within the past 90 days, and (3) consent to participate in the study for 3-4 weeks. Participants were excluded if they: (1) were < 18 years of age at baseline, (2) lived outside the United States, or (3) required wheelchair assistance for most of their daily activities.

Three hundred and eighty-five $(n=385)$ of invited PatientsLikeMe users with MS $(n=$ $1,600)$ responded to the study invitation; 248 respondents met the study criteria based on their responses to the screener via a brief survey. Of the 248 eligible participants that received activity trackers, 203 (82\%) activated the device with the Fitbit website and authorized PatientsLikeMe to access their activity data. Age and gender composition of those that authorized and uploaded data were comparable to those who did not. Those who did not authorize devices and upload data reported higher MSRS score $($ mean $=40)$ than those who authorized devices and uploaded data (mean $=31)$, suggesting that participants with more severe MS were more likely to drop out.

For the secondary analyses, we included data only from participants who: (1) self-reported MSRS within 30 days of their baseline assessment on the PatientsLikeMe website, (2) had complete data on MS type and duration, and (3) produced at least 7 days of wearable device data. The last criterion was based on high data reliability at this level of reporting (Fig. 1).

Data Processing and Transformation

Initial reviews were performed to examine data density, outliers, and unreasonable values. First, day-level physical activity data (step count) were obtained for all participants for the first 25 days in the study. Activity data from the first and last day were removed as these data points may be reflective of a partial day (e.g., participant connects a device the first time on the second half of the day) or may not completely get synchronized or uploaded for the last day. Second, patient-level means and standard deviation of step count were computed for all available days. Day-level data were considered outliers if the values were \pm 3 standard deviation of the patient-level mean. Third, reliability of mean step count data was measured 
Fig. 1. Reliability of wearable measured physical activity (step count): intraclass correlation coefficient (ICC) based on the average number of days $(n=203)$. ICC for an average score = betweensubject variability/(betweensubject variability + within-subject variability/m), where $m$ is the number of measures (days).

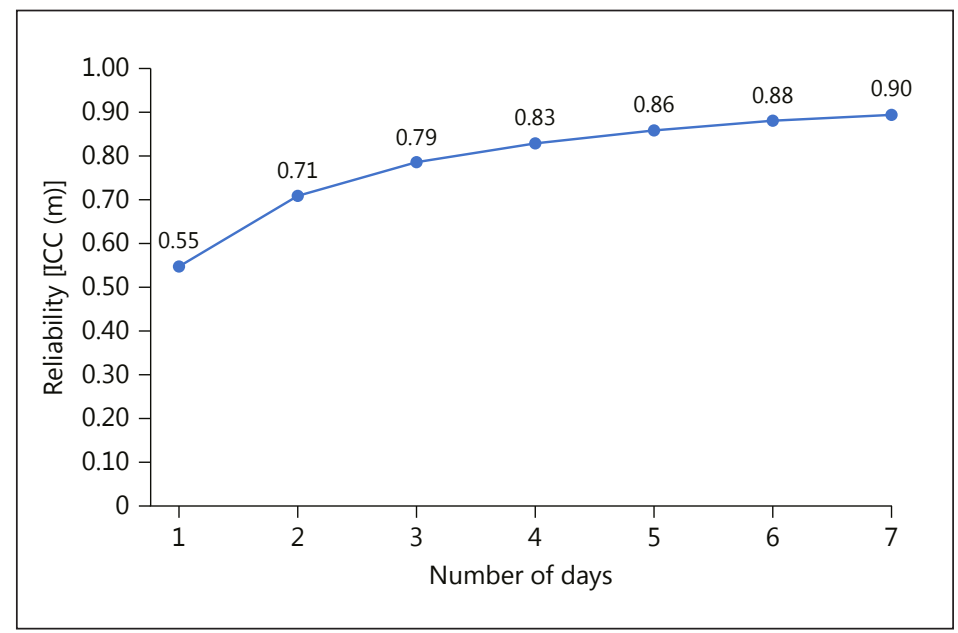

by the intraclass correlation coefficient (ICC) based on repeated-measures analyses of step count data using a one-way random effects model. ICC for a single step count was estimated as the proportion of total variance in the observed measurements that is due to true differences between participants [ICC = between-subject variability/(between-subject variability + within-subject variability)]. The ICC for mean score for more than one measurement was estimated using the Spearman-Brown prophecy formula, which approximates the reliability of a mean measure [ICC = between-subject variability/(between-subject variability + withinsubject variability)/(number of repeated measures)]. Participants with less than 7 days of data were excluded based on an ICC $\geq 0.90$ (excellent reliability) that corresponds to a mean of 7-day measurements. Further, these participants were less compliant and are likely to be sporadic users. The remaining data were used to create a patient-level activity measure (mean, standard deviation, median of step count). Patient-level mean step count was used as a proxy for overall physical activity.

\section{Data Analyses}

Primary Analyses. Descriptive statistics (mean, standard deviation, frequency, percentage) were computed on baseline patient demographic and disease characteristics. Spearman correlation coefficients were computed to examine the association between patient characteristics with physical activity estimates (mean, median step count). Univariate analyses were conducted to test the association between measured physical activity (mean step count) and patient characteristics (age, gender, disease duration, MS type, MSRS). Separate univariate models were constructed with mean step count as the dependent variable and each correlate as the independent variable. Multivariable analyses were constructed with mean step count as the dependent variable and age, gender, disease duration, MS type and last-reported MSRS as the independent variables. Separate multivariable models were constructed with MSRS overall (quartile split) and MSRS walk component score. Univariate and multivariable models were run using the general linear model (PROC GLM) procedure in SAS 9.4 [28]. The type III sum squares were reported, and least square (LS) means were reported as the effect size. Finally, while not directly comparable, physical activity was compared between our MS study population and other general and chronic disease population-based estimates from publicly available data sources [29].

Sensitivity Analyses. These analyses were performed to determine the robustness of the statistical models by examining the extent to which results were affected by changing the model parameters. The changes in the model parameters that were examined were: (a) 
Fig. 2. Flow of participation in the study.

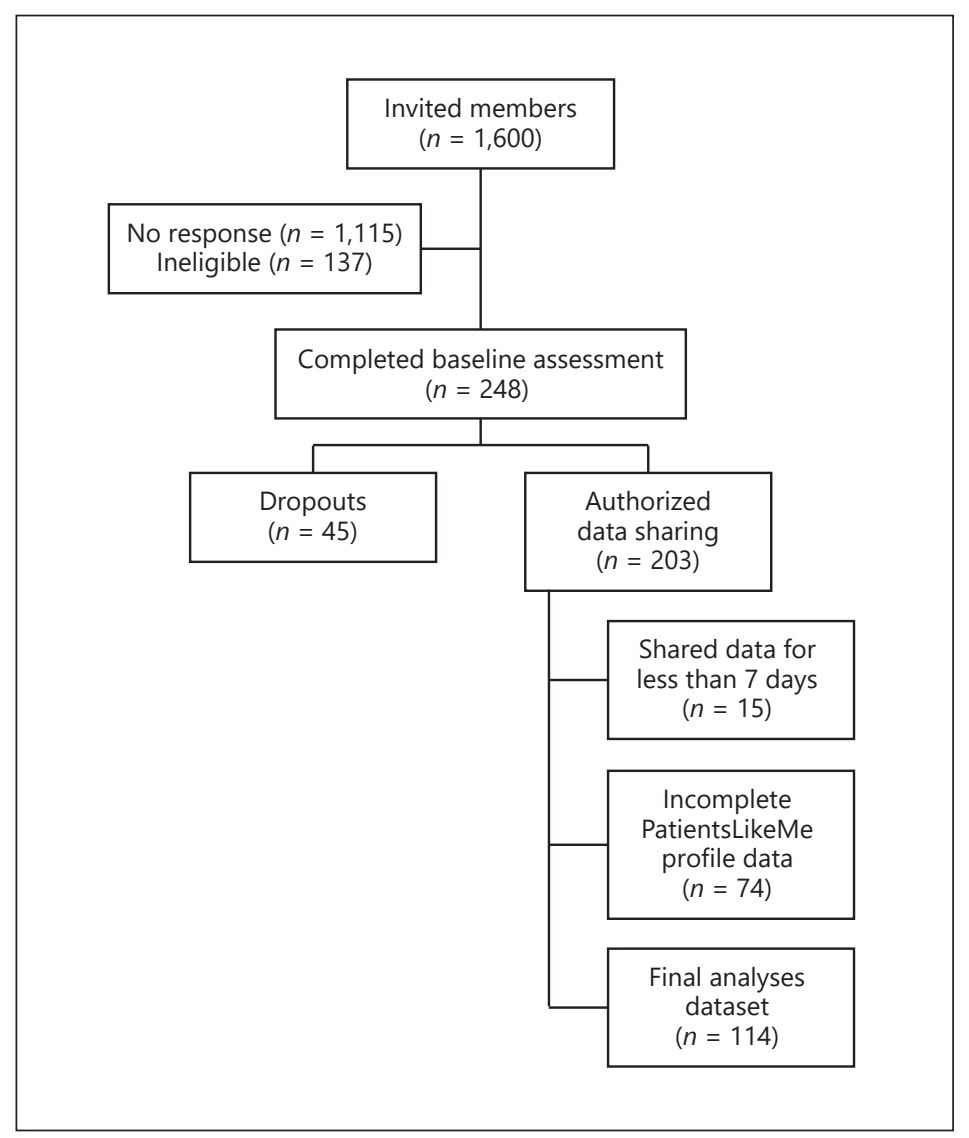

median step count as the outcome (dependent variables), (b) retaining outlier values. All analyses were two-tailed, and the level of significance was set at $\alpha=0.05$. Data analyses were conducted in SAS 9.4 [28].

\section{Results of Secondary Analysis}

\section{Participants}

After excluding participants with less than 7 days of data upload and participants with missing data on MSRS and disease duration, the final sample for secondary data analyses consisted of 114 participants (Fig. 2).

In the final cohort, participants uploaded a mean 20.1 days of data over the 23-day study (87\% adherence); most uploaded data for 14 days or more (92\%); 68\% uploaded data for 21 days or more (Fig. 2). The sample mean of estimated mean daily step count was 4,393 (SD = $2,603)$, and the median was 4,274 (SD = 2,791).

The mean age of eligible participants was 52 years ( $S D=9)$, the majority were female (75\%), relapsing-remitting type (79\%) with a mean disease duration of 16 years (SD $=10)$. MSRS score within 30-day of baseline was $32(\mathrm{SD}=16)$ with $72 \%$ reporting mild-moderate walking disability (Table 1).

\section{Reliability of Wearable Measured Physical Activity (Step Count)}

Analyses illustrated in Figure 1 show that the reliability estimates of the average of step count as measured by ICC increased with more days of data. Reliability for a single day step 
Table 2. Spearman correlation coefficients of activity measures and correlates

\begin{tabular}{llllll}
\hline & $\begin{array}{l}\text { Disease } \\
\text { duration }\end{array}$ & $\begin{array}{l}\text { MSRS } \\
\text { (past 30 days) }\end{array}$ & $\begin{array}{l}\text { MSRS walk score } \\
\text { (past 30 days) }\end{array}$ & $\begin{array}{l}\text { Mean } \\
\text { step count }\end{array}$ & $\begin{array}{l}\text { Median step } \\
\text { count }\end{array}$ \\
\hline Age & 0.43 & 0.01 & 0.15 & 0.01 & 0.02 \\
& $p<0.001$ & $p=0.94$ & $p=0.12$ & $p=0.90$ & $p=0.80$ \\
\hline Disease duration & & 0.09 & 0.19 & 0.00 & -0.01 \\
& $p=0.32$ & $p<0.05$ & $p=0.98$ & $p=0.93$ \\
\hline MSRS (past 30 days) & & 0.70 & -0.40 & -0.41 \\
& & $p<0.001$ & $p<0.001$ & $p<0.001$ \\
\hline MSRS walk score (past 30 days) & & & -0.39 & -0.37 \\
& & & & $p<0.001$ & $p<0.001$ \\
\hline Mean step count & & & & 0.99 \\
& & & & $p<0.001$ \\
\hline
\end{tabular}

Table 3. Correlates of free-living physical activity measured by mean step count for those that report MSRS within the past 30 days

\begin{tabular}{|c|c|c|c|c|c|c|}
\hline & \multicolumn{2}{|c|}{$\begin{array}{l}\text { Univariate model } \\
(n=114)\end{array}$} & \multicolumn{2}{|c|}{$\begin{array}{l}\text { Multivariable model I } \\
(n=114)\end{array}$} & \multicolumn{2}{|c|}{$\begin{array}{l}\text { Multivariable model II } \\
(n=114)\end{array}$} \\
\hline & LS means & $p$ value & LS means & $p$ value & LS means & $p$ value \\
\hline Age & & 0.82 & & 0.43 & & 0.42 \\
\hline 18-39 years & 3,885 & & 3,583 & & 3,051 & \\
\hline $40-49$ years & 4,586 & & 4,680 & & 4,570 & \\
\hline 50-59 years & 4,528 & & 4,655 & & 4,611 & \\
\hline 60 years or more & 4,119 & & 3,907 & & 4,271 & \\
\hline Gender & & 0.50 & & 0.17 & & 0.42 \\
\hline Male & 4,681 & & 4,967 & & 4,728 & \\
\hline Female & 4,299 & & 4,206 & & 4,284 & \\
\hline Disease duration & & 0.99 & & 0.71 & & 0.66 \\
\hline Less than 5 years & 4,402 & & 4,482 & & 4,748 & \\
\hline $5-9$ years & 4,442 & & 4,037 & & 4,037 & \\
\hline 10 years or more & 4,372 & & 4,520 & & 4,476 & \\
\hline MSRS (last reported within past 30 days) & & $<0.001$ & & $<0.001$ & - & - \\
\hline 1st quartile (least disability) & 5,795 & & 5,937 & & & \\
\hline 2nd quartile & 4,571 & & 4,570 & & & \\
\hline 3rd quartile & 3,646 & & 3,490 & & & \\
\hline 4th quartile (highest disability) & 3,259 & & 3,272 & & & \\
\hline MSRS walk score (last reported within & & & & & & \\
\hline past 30 days) & & $<0.001$ & - & - & & $<0.001$ \\
\hline No symptom, no disability & 6,639 & & & & 6,931 & \\
\hline Symptom, no disability & 4,820 & & & & 4,743 & \\
\hline Mild disability & 4,460 & & & & 4,394 & \\
\hline Moderate disability & 2,613 & & & & 2,727 & \\
\hline MS type & & 0.09 & & 0.17 & & 0.51 \\
\hline Relapsing remitting & 4,666 & & 4,630 & & 4,539 & \\
\hline Secondary progressive & 3,398 & & 3,392 & & 3,711 & \\
\hline Primary progressive & 3,329 & & 3,659 & & 4,035 & \\
\hline
\end{tabular}

Analyses conducted using general linear model (GLM) with mean step count as the dependent variable. Univariate analyses were conducted by fitting separate GLM models for each correlate. Model I included age, gender, disease duration, last-reported MSRS within past 30 days (quartile), and MS type as independent variables. Model II included age, gender, disease duration, last-reported MSRS walk score within past 30 days, and MS type as independent variables. LS, least square. 
DasMahapatra et al.: Free-Living Wearable Activity Monitoring in Multiple Sclerosis

LS means of participants that were least disabled (lowest MSRS quartile) was 5,937 steps, which was significantly higher than the second, third, and fourth quartiles $(4,570,3,490$, and 3,272 steps, respectively) (Table 3). Similarly, estimated LS means of participants with no ambulatory disability (measured by MSRS walk component) was 6,931 steps, which is significantly higher compared to participants with greater disability. Age, gender, disease duration and MS type were not independently associated with mean step count.

Although not statistically significant, participants with relapsing-remitting MS were more active than those with secondary progressive and primary progressive MS. This may not always be the case, since some early stage secondary and primary progressive MS patients may be more active than relapsing-remitting MS patients. Further, given that relapses manifest with clinical worsening of symptoms, it is likely that relapsing-remitting patients will have greater within-subject variability in mobility.

\section{Sensitivity Analyses}

Sensitivity analyses were performed to determine the robustness of the statistical models by examining the extent to which results were affected by changing the model parameters. Sensitivity analyses revealed that the results were consistent after changing the model parameters to: (a) median step count as the outcome (dependent variables), and (b) retaining outlier values.

\section{Physical Activity Relative to General and Other Chronic Disease Populations}

While not directly comparable, reports from other publicly available studies indicate that our cohort of MS patients may be slightly less physically active compared to the general population but more active comparable to older patients with obstructive pulmonary disease (Table 4).

Participants in the 18-39 age group were less physically active than those in their fifth and sixth decades of life. Participants in the 60+ age group were also less physically active (Fig. 3). It should be noted that the studies cited differed with respect to the design, duration of observation, type of device used, and demographic composition compared to this study.

\section{Discussion}

The current study aimed to replicate and expand upon the published literature on the utility of free-living physical activity monitoring in MS. While physical activity measured by wearable devices has potential applications in characterizing, monitoring patients, and designing interventions, robust analytics on wearable device data is dependent on two critical factors: (1) successful adoption and compliance by patients and (2) reduction in the measurement error. For example, in a real-world setting, observations from some patients can reflect an erroneous characterization of true activity due to infrequent or sporadic use [31]. Further, physical activity is highly variable from day to day, and some studies have shown that activity patterns can vary by the day of the week and patient type [32].

This 3-week study revealed five major findings. First, averaging 2 days of step counts provides an adequate level of reliability $(\geq 0.7)$, which approximates a very high level of reliability estimates when averaging data for an entire week (0.9). This was replicated by Norris et al. [23], who reported similar findings using an approach that examined reliability coefficients from random 2-day to 7-day averages. In addition, obtaining a stable measure of freeliving walking activity based on pedometer use may require 7 days in healthy individuals [33], while more impaired populations such as incomplete spinal cord injury patients may require only 2 days of monitoring to achieve acceptably stable estimates [34]. This finding has practical implications with respect to utilizing wearable generated activity data in clinical 

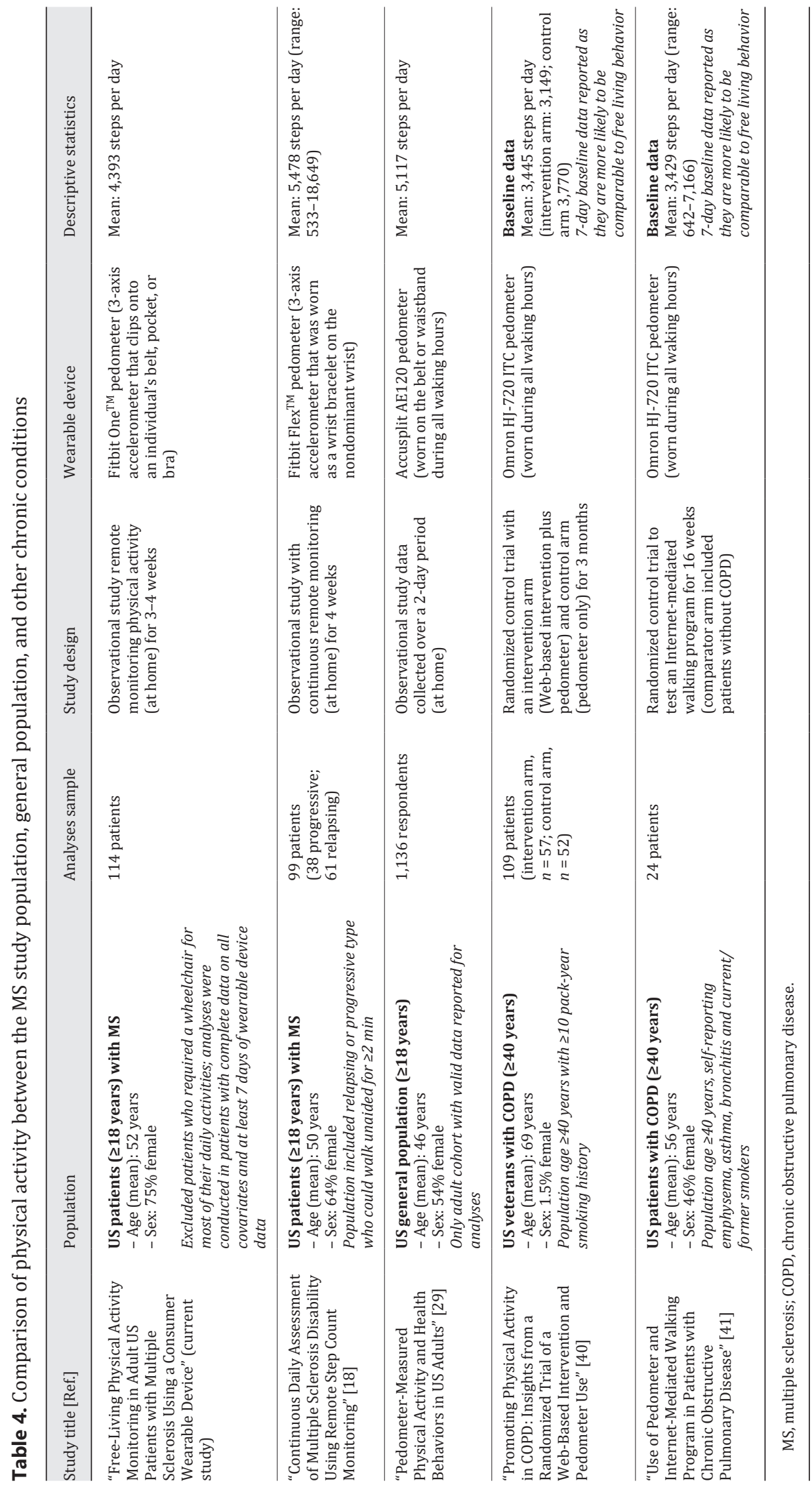


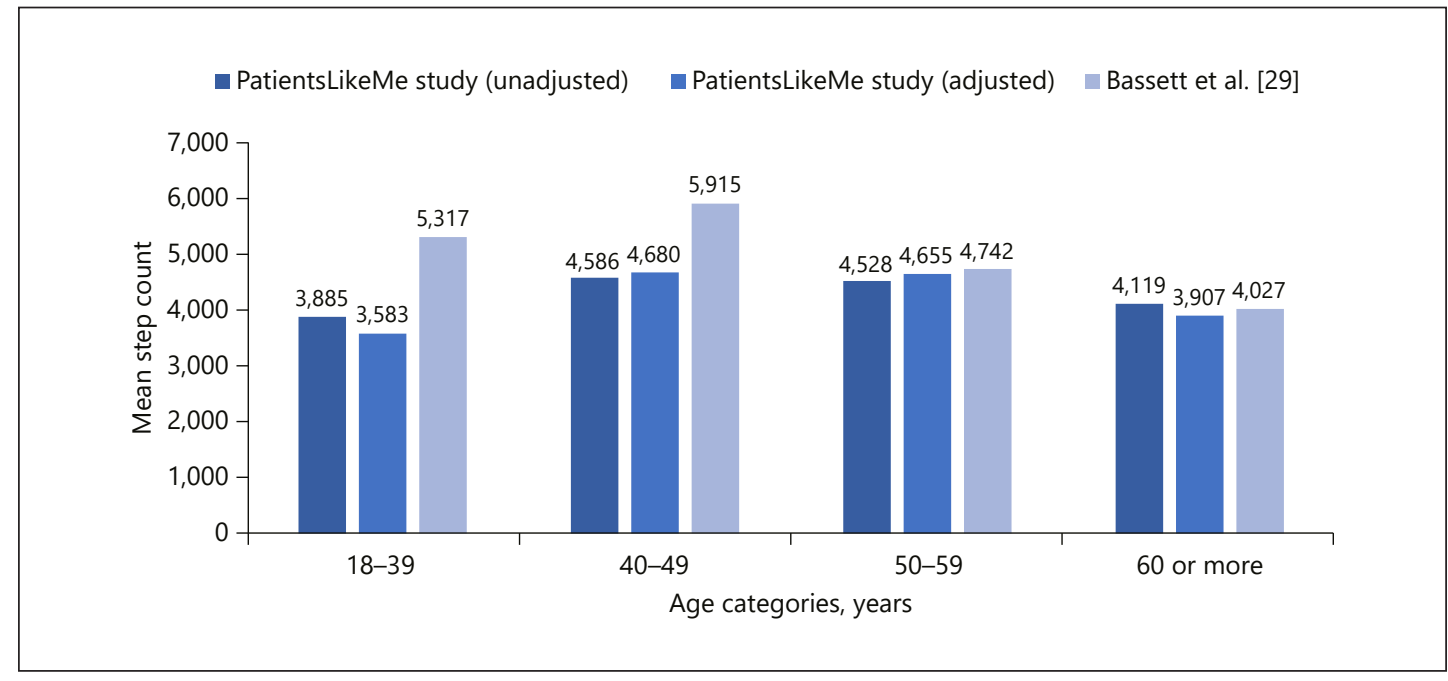

Fig. 3. Comparison of age-related change in activity between the MS study population and the general population. Adjusted for gender, disease duration, last-reported Multiple Sclerosis Rating Scale score within past 30 days (quartile), and MS type.

research. For example, the overall real-world status of a patients' mobility can be determined by obtaining data for about a week instead of continuous monitoring which minimizes the burden on the patient.

It is noteworthy that despite the ability to provide high-resolution quantitative data, these devices have specific limitations for widespread use in the clinical setting [35]. Among other factors, the need for a high rate of patient compliance with wearing the device all the time affects the use of data from pedometers and accelerometers. To be practical, for device use in the regular assessment of patients with MS, it is critical to reduce measurement error. Such a reduction in measurement error improves the ability to detect change and also improves the mathematical models to detect whether patients with true systematic differences can be distinguished from each other. Investigators have adopted several data management steps to reduce measurement error which include: (a) excluding data from users that do not use the device above a threshold value (e.g., $\geq 3$ days/week, $\geq 8 \mathrm{~h} /$ day), (b) removing outlier data, and (c) averaging values for multiple days to improve reliability coefficients [32]. These guidelines are generally acceptable to improve data quality and better characterize patients' ambulation.

Second, the characterization of participants based on activity patterns by age, gender, disease duration, and MS type did not uncover statistically significant independent associations with step count but directionally conformed to clinical presentations. For example, participants with relapsing-remitting MS were more active than participants with progressive MS. It should be noted, however, that relapsing events were not evaluated in the study. Given that relapses manifest with clinical worsening of symptoms, free-living activity monitoring has the potential of detecting the fluctuations in mobility during and between replacing events. Taken together, these findings suggest that physical active trackers can be applied to characterizing patient types based on their ambulatory function.

Third, we observed an independent association between subjective assessment of MS-disability, the MSRS, and objective data (steps count). Among several variables studied, self-reported disability was an independent predictor of mean step count. Low step count was associated with increased disability. This finding replicates previous findings of strong 
DasMahapatra et al.: Free-Living Wearable Activity Monitoring in Multiple Sclerosis

correlations between self-report and accelerometry measures for MS [36]. Studies have shown similar correlations between sensor data streams and functional rating scales for other neurological conditions, suggesting this could be an area for further exploration [16].

In addition, participants with no walking symptoms or disability averaged more than 2,000 steps per day compared to those that experience symptoms but did not have a disability, suggesting that the onset of symptom may be a driver of diminished physical activity. Similarly, studies that used MSWS and PDDS as anchor points to activity measures have found clinically meaningful change with a difference of 800 steps per day [36]. Future studies are warranted to evaluate the responsiveness of change in step counts in relation to change in their disease severity and disability.

Fourth, comparisons of age-related change in activity in the MS population relative to the general population and other disease cohorts indicate that our cohort of MS participants was slightly less physically active compared to the general population and comparable to patients on cardiac rehabilitation [37]. On comparing our study to a large population-based study in the general population [29], it is noteworthy that while the measured mean step count in our study was lower, the patterns in activity change with age were similar. Another cohort of MS was found to be more physically active, although the study included only 11 participants that were able to walk independently [4]. As expected, Motl et al. [38] found that MS patients were less physically active than adults without MS.

Finally, our engagement model offers an alternative to traditional clinical site-based studies and is worth consideration for future research. In contrast to time-intensive clinical studies, a large sample of patients was recruited in less than a day from several states in the US. Outreaching an online network of research-ready patients and providing access to an interactive platform enabled accelerated recruitment and efficient data collection on disease correlates and freeliving mobility. Merits and demerits of this approach should be evaluated in future studies.

Studies in the last decade have laid the foundation for wearable technologies to support patients, clinicians, and the industry in MS. Patients can easily access inexpensive, attractive and user friendly consumer wearables to monitor their health and disease progression. Clinicians can use the technology to get a better understanding of the patients' health in consultations. Further, such tools have the potential to support clinical decisions by identifying patients at risk of relapse and requiring interventions. A recent study in cystic fibrosis patients is piloting digital spirometers to identify patients at risk of exacerbations. For the industry, wearables offer a novel method of evaluating clinical benefit of treatment in the real-world setting. At the time of evolution of data science, advanced analytical tools, and artificial intelligence, the future of wearable technology in MS assessment and care is promising.

\section{Limitations}

The current study has several limitations. First, the original study was intended as a pilot study with limited data collected on the socioeconomic background, physical status, normal ambulation environment, behaviors, and demographic status of participants. Multivariable analyses were limited to data that were collected as part of the study and not all correlates on mobility in MS. Second, given the short period of data collection, continued assessment of these participants' change over time was not performed. To our knowledge, at the time of this publication, no wearable study has successfully detected the progression of MS over time as a result of the challenges in longitudinal data collection. The engagement framework established in this study may facilitate large-scale recruitment and longitudinal data collection in patient-powered communities for future studies. Third, pedometer-based step counting does not characterize all movement disturbances (e.g., gait) in participants with MS [17]. Fourth, it is not possible to determine whether patient motivation/activation would be any different for populations who are less engaged in health information sharing. The comparisons with 
DasMahapatra et al.: Free-Living Wearable Activity Monitoring in Multiple Sclerosis

other studies are also potentially confounded by demographic differences, period of observation, behavioral reactivity, study design, and type of device used. Fifth, participants that opt into such patient platforms are predominantly female, more engaged, and technologically aware than most patients. The findings may not be generalizable to the MS population at large. The PatientsLikeMe MS population, however, is quite representative of the overall MS population [39]. Finally, a significant limitation is the absence of clinical and physiciangenerated data, e.g. Multiple Sclerosis Functional Composite (MSFC), provider-determined EDSS, MRI metrics, etc. The diagnosis is also patient reported. It should be noted, however, that another study conducted in a sample of MS patients in the PatientsLikeMe database found high concordance in diagnosis of MS when compared to an insurance claims database, supporting the validity of self-reported diagnosis as clinically diagnosed MS [26]. While this study did not intend to solicit physician-generated data, the absence must be acknowledged as an important limitation when interpreting the results.

\section{Conclusion}

In conclusion, this study advanced the current literature by engaging a range of MS patients that are members of an online research network, leveraging an interactive platform to collect Fitbit data remotely. Our engagement model offers an alternative to traditional clinical site-based studies. The use of an online network allowed rapid recruitment, integration of activity data with an existing data set containing demographics, disease symptoms, and treatments, and longitudinal patient involvement with research. The leveraging of such technology will be critical in efficiently capturing user activity metrics in real-world settings.

In addition, important metrics such as the use of a minimum of 2-day estimates and selfreported disability were found to be robust indicators and correlates, respectively, of participant activity levels. Because MS patients may be inconsistent in the use of devices as is seen in other populations, there will be a need to establish good estimates of activity in less than optimal conditions. Further exploration and triangulation of such metrics may reduce the burden on patients, clinicians, and researchers when monitoring clinical status.

\section{Acknowledgement}

We are grateful to the MS patient members of PatientsLikeMe for volunteering to participate in this study, Paul Wicks for recommendations regarding conceptual development and content in the paper, and Chris Curran for data analysis support.

\section{Statement of Ethics}

Independent ethics review was sought, and the study protocol received ethical approval from the New England Institutional Review Board (NEIRB) on June 6, 2014.

\section{Disclosure Statement}

At the time of manuscript preparation, P.D. was an employee of PatientsLikeMe and owns stock options in the company. E.C. and R.B. are employees of PatientsLikeMe and own stock options in the company. The PatientsLikeMe Research Team has received research funding 
(including conference support and consulting fees) from AbbVie, Accorda, Actelion, Amgen, AstraZeneca, Avanir, Biogen, Boehringer Ingelheim, Genzyme, Genentech, Janssen, Johnson \& Johnson, Merck, Novartis, Pfizer, Sanofi, Takeda, and UCB. The PatientsLikeMe R\&D team has received research grant funding from Kaiser Permanente, the Robert Wood Johnson Foundation, Sage Bionetworks, The AKU Society, and the University of Maryland. At the time of the manuscript submission, J.R. was an employee of Biogen and owns stock options in the company.

\section{Funding Sources}

This study was funded by Biogen.

\section{Author Contributions}

P.D. was involved in the conceptualization, contributed to the writing and revision, conducted data analysis, approved final content, and is accountable for all aspects of the work. E.C. was involved in the conceptualization, contributed to the writing and revision, reviewed the data analysis, approved final content of the paper, and is accountable for all aspects of the work. R.B. was involved in the conceptualization, contributed to the revision, reviewed the data analysis, approved final content of the paper, and is accountable for all aspects of the work. J.R. was involved in conceptualization, contributed to the revision, approved final content of the paper, and is accountable for all aspects of the work.

\section{Appendix}

The Multiple Sclerosis Rating Scale (MSRS) as Presented to Members of the PatientsLikeMe MS Community

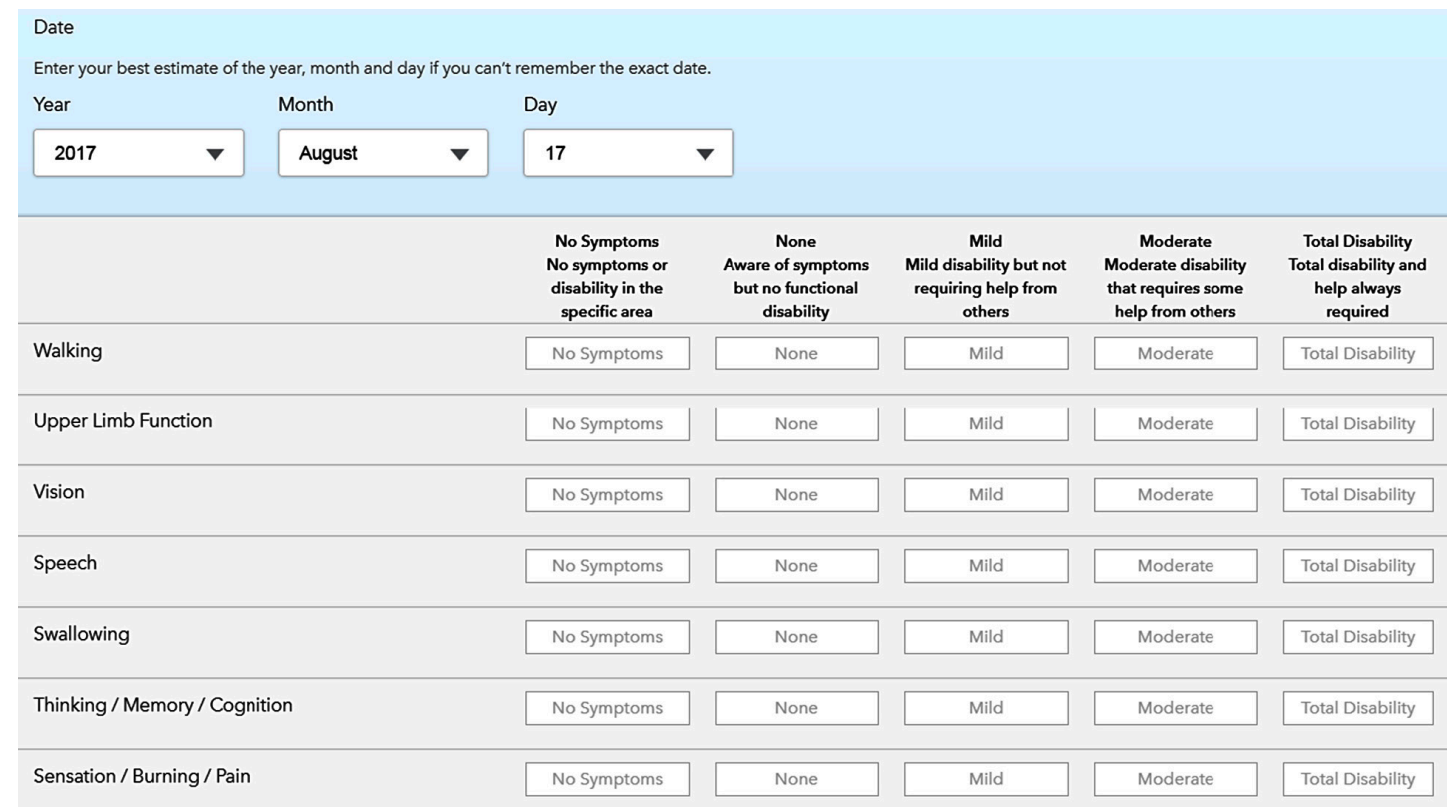




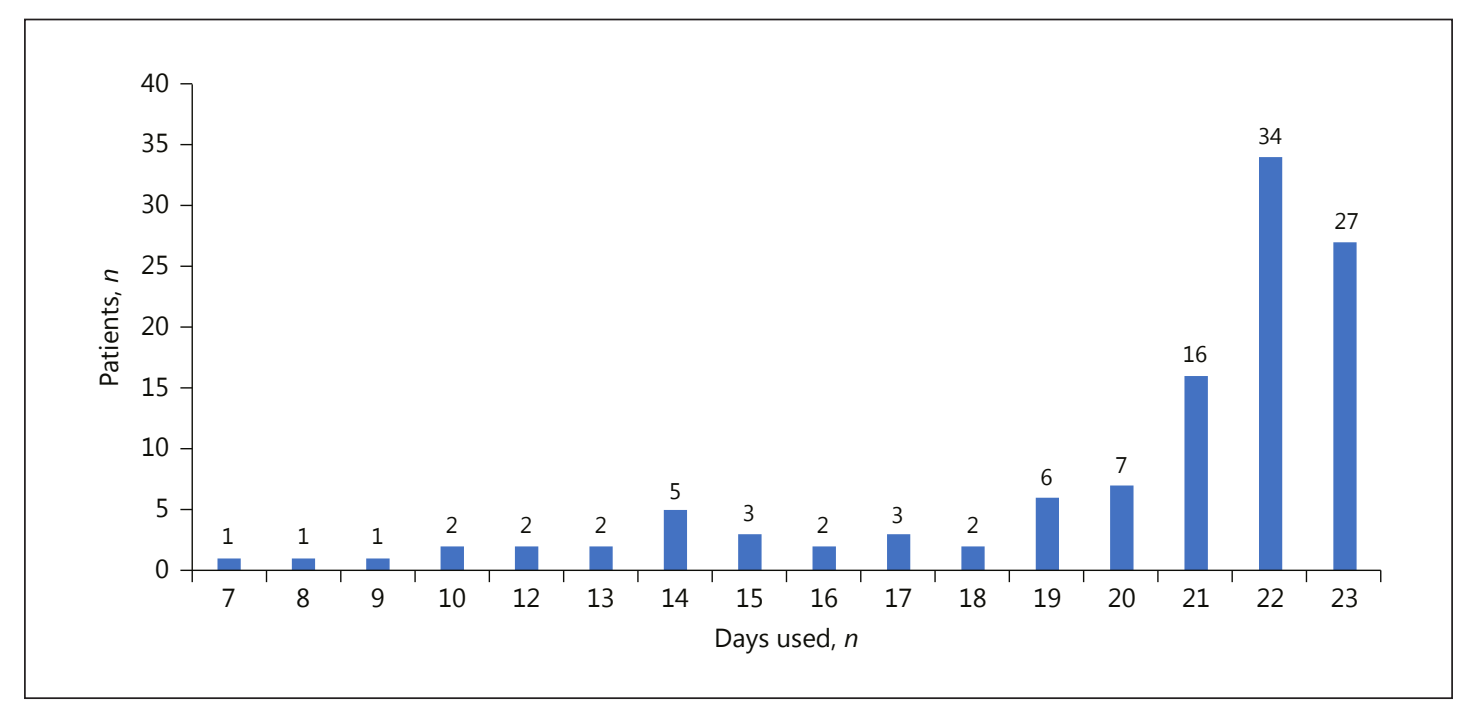

\section{References}

1 Piwek L, Ellis DA, Andrews S, Joinson A: The rise of consumer health wearables: promises and barriers. PLoS Med 2016;13:e1001953.

2 FDA: Digital Health. Washington, US Department of Health and Human Services, 2017. https://www.fda.gov/ MedicalDevices/DigitalHealth/.

3 Warburton DER, Nicol CW, Bredin SSD: Health benefits of physical activity: the evidence. Can Med Assoc J 2006; 174:801-809.

4 Shammas L, Zentek T, von Haaren B, Schlesinger S, Hey S, Rashid A: Home-based system for physical activity monitoring in patients with multiple sclerosis (pilot study). Biomed Eng Online 2014;13:10.

5 Paton C, Hansen Edd MM, Fernandez-Luque L, Lau A: Self-tracking, social media and personal health records for patient empowered self-care. 2012. http://repository.usfca.edu/nursing_fac.

6 Lauritzen J, Muñoz A, Sevillano JL, Civit A: The Usefulness of Activity Trackers in Elderly with Reduced Mobility: A Case Study. Amsterdam, IOS Press Ebooks, 2013. http://ebooks.iospress.nl/publication/34101 (cited March 22, 2017).

7 Hills AP, Mokhtar N, Byrne NM: Assessment of physical activity and energy expenditure: an overview of objective measures. Front Nutr 2014;1:5.

8 Beg MS, Gupta A, Stewart T, Rethorst CD: Promise of wearable physical activity monitors in oncology practice. J Oncol Pract 2017;13:82-89.

9 Evenson KR, Goto MM, Furberg RD: Systematic review of the validity and reliability of consumer-wearable activity trackers. Int J Behav Nutr Phys Act 2015;12:159.

10 Wong CK, Mentis HM, Kuber R: The bit doesn't fit: evaluation of a commercial activity-tracker at slower walking speeds. Gait Posture 2018;59:177-181.

11 Chiauzzi E, Rodarte C, DasMahapatra P: Patient-centered activity monitoring in the self-management of chronic health conditions. BMC Med 2015;13:77.

12 Maher C, Ryan J, Ambrosi C, Edney S: Users' experiences of wearable activity trackers: a cross-sectional study. BMC Public Health 2017;17:880.

13 Birkeland K, Khandwalla RM, Kedan I, Shufelt CL, Mehta PK, Minissian MB, et al: Daily activity measured with wearable technology as a novel measurement of treatment effect in patients with coronary microvascular dysfunction: substudy of a randomized controlled crossover trial. JMIR Res Protoc 2017;6:e255.

14 Vaes AW, Cheung A, Atakhorrami M, Groenen MTJ, Amft O, Franssen FME, et al: Effect of "activity monitorbased" counseling on physical activity and health-related outcomes in patients with chronic diseases: a systematic review and meta-analysis. Ann Med 2013;45:397-412.

15 Yousef A, Jonzzon S, Suleiman L, Arjona J, Graves JS: Biosensing in multiple sclerosis. Expert Rev Med Devices 2017;14:901-912.

16 Block VAJ, Pitsch E, Tahir P, Cree BAC, Allen DD, Gelfand JM: Remote physical activity monitoring in neurological disease: a systematic review. PLoS One 2016;11:e0154335.

17 Bethoux F, Bennett S: Evaluating walking in patients with multiple sclerosis. Int J MS Care 2011;13:4-14.

18 Block VJ, Lizée A, Crabtree-Hartman E, Bevan CJ, Graves JS, Bove R, et al: Continuous daily assessment of multiple sclerosis disability using remote step count monitoring. J Neurol 2017;264:316-326. 


\begin{tabular}{|c|c|}
\hline \multicolumn{2}{|l|}{ Digit Biomark 2018;2:47-63 } \\
\hline DOI: $10.1159 / 000488040$ & $\begin{array}{l}\text { (c) } 2018 \text { The Author(s). Published by S. Karger AG, Basel } \\
\text { www.karger.com/dib }\end{array}$ \\
\hline
\end{tabular}

DasMahapatra et al.: Free-Living Wearable Activity Monitoring in Multiple Sclerosis

19 Busse ME, Pearson OR, Van Deursen R, Wiles CM: Quantified measurement of activity provides insight into motor function and recovery in neurological disease. J Neurol Neurosurg Psychiatry 2004;75:884-888.

20 Motl RW, Sandroff BM, Sosnoff JJ: Commercially available accelerometry as an ecologically valid measure of ambulation in individuals with multiple sclerosis. Expert Rev Neurother 2012;12:1079-1088.

21 Dlugonski D, Pilutti LA, Sandroff BM, Suh Y, Balantrapu S, Motl RW: Steps per day among persons with multiple sclerosis: variation by demographic, clinical, and device characteristics. Arch Phys Med Rehabil 2013;94: 1534-1539.

22 Motl RW, Pilutti LA, Learmonth YC, Goldman MD, Brown T: Clinical importance of steps taken per day among persons with multiple sclerosis. PLoS One 2013;8:e73247.

23 Norris M, Anderson R, Motl RW, Hayes S, Coote S: Minimum number of days required for a reliable estimate of daily step count and energy expenditure, in people with MS who walk unaided. Gait Posture 2017;53:201206.

24 McIninch J, Datta S, DasMahapatra P, Chiauzzi E, Bhalerao R, Spector A, et al: Remote tracking of walking activity in MS patients in a real-world setting (P3.209). Neurology 2015;84(14 suppl):P3.209.

25 Brownstein CA, Brownstein JS, Williams DS, Wicks P, Heywood JA: The power of social networking in medicine. Nat Biotechnol 2009;27:888-890.

26 Eichler GS, Cochin E, Han J, Hu S, Vaughan TE, Wicks P, et al: Exploring concordance of patient-reported information on PatientsLikeMe and medical claims data at the patient level. J Med Internet Res 2016;18:e110.

27 Bove R, Secor E, Healy BC, Musallam A, Vaughan T, Glanz BI, et al: Evaluation of an online platform for multiple sclerosis research: patient description, validation of severity scale, and exploration of BMI effects on disease course. PLoS One 2013;8:e59707.

28 SAS 9.4. Cary, NC, USA: Editorial Guidelines. https://www.sas.com/en_us/legal/editorial-guidelines.html (cited September 27, 2017).

29 Bassett DR, Wyatt HR, Thompson H, Peters JC, Hill JO, Hill JO: Pedometer-measured physical activity and health behaviors in U.S. adults. Med Sci Sports Exerc 2010;42:1819-1825.

30 Cappelleri JC, Zou KH, Bushmakin AG, Alvir JMJ, Alemayehu D, Symonds T: Patient-reported Outcomes: Measurement, Implementation and Interpretation, ed 1. Boca Raton, CRC Press, 2014.

31 Chiauzzi E, Rodarte C, DasMahapatra P: Patient-centered activity monitoring in the self-management of chronic health conditions. BMC Med 2015;13:77.

32 Evenson KR, Wen F, Metzger JS, Herring AH: Physical activity and sedentary behavior patterns using accelerometry from a national sample of United States adults. Int J Behav Nutr Phys Act 2015;12:20.

33 Hale LA, Pal J, Becker I: Measuring free-living physical activity in adults with and without neurologic dysfunction with a triaxial accelerometer. Arch Phys Med Rehabil 2008;89:1765-1771.

34 Ishikawa S, Stevens S, Kang M, Morgan D: Reliability of daily step activity monitoring in adults with incomplete spinal cord injury. J Rehabil Res Dev 2011;48:1187-1194.

35 Pearson OR, Busse ME, van Deursen RWM, Wiles CM: Quantification of walking mobility in neurological disorders. QJM 2004;97:463-475.

36 Snook EM, Motl RW, Gliottoni RC: The effect of walking mobility on the measurement of physical activity using accelerometry in multiple sclerosis. Clin Rehabil 2009;23:248-258.

37 Cupples M, Dean A, Tully MA, Taggart M, McCorkell G, Neill SO, et al: Using pedometer step-count goals to promote physical activity in cardiac rehabilitation: a feasibility study of a controlled trial. Int J Phys Med Rehabil DOI: 10.4172/2329-9096.1000157.

38 Motl RW, McAuley E, Klaren R: Reliability of physical-activity measures over six months in adults with multiple sclerosis: implications for designing behavioral interventions. Behav Med 2014;40:29-33.

39 Wicks P, Vaughan TE, Massagli MP: The multiple sclerosis rating scale, revised (MSRS-R): development, refinement, and psychometric validation using an online community. Health Qual Life Outcomes 2012;10:70.

40 Wan ES, Kantorowski A, Homsy D, Teylan M, Kadri R, Richardson CR, Gagnon DR, Garshick E, Moy ML: Promoting physical activity in COPD: insights from a randomized trial of a Web-based intervention and pedometer use. Respir Medicone 2017;130:102-110.

41 Moy ML, Janney AW, Nguyen HQ, et al: Use of pedometer and Internet-mediated walking program in patients with chronic obstructive pulmonary disease. J Rehabil Res Dev 2010;47:485-496. 